\title{
Prevalence of Amblyopia and Associated Risk Factors in Tibetan Grade One Children
}

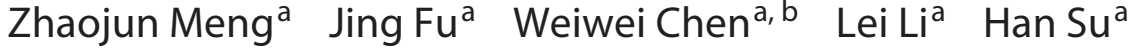 \\ Wei Dai ${ }^{\mathrm{a}}$ Yao Yao ${ }^{\mathrm{a}}$ \\ aBeijing Tongren Eye Center, Beijing Tongren Hospital, Capital Medical University, \\ Beijing Ophthalmology \& Visual Sciences Key Laboratory, Beijing, China; beijing Institute of \\ Ophthalmology, Capital Medical University, Beijing, China
}

\section{Keywords}

Amblyopia $\cdot$ Prevalence $\cdot$ Risk factors $\cdot$ Ophthalmic epidemiology $\cdot$ Clinical trial

\begin{abstract}
Introduction: Amblyopia is an important public health problem and standard screening is quite necessary for early diagnosis and treatment especially for the remote areas. As the place of the largest Tibetan population, the Tibetan Plateau has special geographical characteristics such as high altitude, time zone, and ethnic composition, where very little information is available about the prevalence of amblyopia and other ocular diseases. The article aims to determine the prevalence of amblyopia and associated factors in grade 1 Tibetan children, living in Lhasa, Tibet Autonomous Region. Methods: A cross-sectional study was conducted. All the participants were scheduled for comprehensive eye examinations including visual acuity testing, ocular deviation and movement evaluation, cycloplegic refraction and examinations of the external eye, anterior segment, media, and fundus. Amblyopia was assessed in the children according to the standard definition. Results: A total of 1,856 stu-
\end{abstract}

dents participated in the examinations $(97.58 \%$ response rate). 1,852 students completed all the related examinations, and 34 of them were diagnosed as amblyopia with the prevalence of $1.84 \%$ (95\% confidence interval [Cl]: $1.22-$ $2.45 \%)$. Unilateral amblyopia was diagnosed in 23 students (1.24\%, 95\% Cl: 0.74-1.75\%), including 16 anisometropic, 4 strabismic, 1 visual deprivational, and 2 mixed. Other 11 students were diagnosed as bilateral amblyopia (0.59\%, 95\% Cl: 0.24-0.94\%), including 9 ametropic, 1 deprivational, and 1 with nystagmus. The mean cylinder refraction and absolute value of the spherical equivalent refraction of amblyopic eyes was respectively $-2.15 \pm 1.52 \mathrm{D}$ and $2.70 \pm 2.33 \mathrm{D}$. Amblyopia was significantly associated with hyperopia $(\geq+2.00 \mathrm{D}$, odds ratio [OR] 8.22, 95\% Cl 3.42-19.72), astigmatism ( $\leq-2.00 \mathrm{D}$, OR $6.76,95 \% \mathrm{Cl} 2.56-17.85)$, and anisometropia ( $\geq+0.50$ to $<+1.00$ D, OR 3.95, 95\% Cl 1.44-10.79; $\geq+1.00$ D, OR 21.90, 95\% Cl 8.24-58.18). Conclusions: The prevalence of amblyopia in grade 1 students of Lhasa is relatively higher than that of many other ethnic populations in China previously reported. Refractive errors including anisometropia, hyperopia, and astigmatism are the major risk factors of amblyopia.

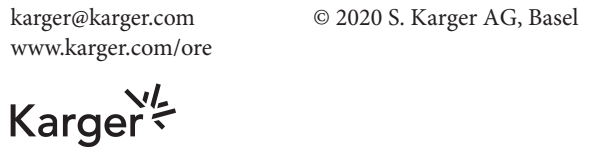




\section{Introduction}

Amblyopia is an important public health problem because its visual impairment can be lifelong and profound [1-3]. The latest meta-analysis article estimated 99.2 million people with amblyopia in 2019 worldwide, increasing to 175.2 million by 2030 and 221.9 million by 2040 [4]. Understanding the prevalence rate is important for adequate healthcare planning. Substantial studies have been developed about the prevalence of amblyopia and the results vary considerably from 1 to $6 \%$ in children [5-9] and $1.43-5.64 \%$ in adults $[10,11]$. This range is considered to be influenced by age, region, ethnicity, diagnosis criteria, and other factors.

In China there have been several studies about the prevalence of amblyopia from discrete regions, such as the Anyang Childhood Eye Study (ACES), the Nanjing Pediatric Vision Project (NPVP), and so on which reported the rates ranging from 0.82 to $1.47 \%$ [12-16]. These studies mainly focus on the Han, accounting for $91.5 \%$ of the whole Chinese population, from the plain area. Nevertheless, very little information is available about the prevalence of amblyopia in children from Tibetan areas at high altitude. The variations of ethnicity, environment factors such as the climate, light, living habits, and many other factors can potentially affect the prevalence. So evaluating the prevalence of amblyopia in Tibetan areas is of great significance and is also an important supplement to other population studies in China and the world.

The Lhasa Childhood Eye Study (LCES) is conducted in the center of Tibetan plateau in Southwest China and will last from 2019 to 2024 . It aims to research the incidence and progression of major ocular diseases affecting Chinese Tibet Plateau children. It is significant to develop the study among grade 1 students in view of their sensitive period of vision development, the relatively mature cognitive abilities and the better follow-up. As the baseline part of the LCES, the main purpose of current study was to determine the prevalence, causes, and associations of amblyopia in grade 1 Tibetan children.

\section{Methods}

\section{Study Design and Population}

The LCES is a school-based cohort study designed to observe the occurrence and development of different ocular diseases and will last from 2019 to 2024. The study adhered to the Declaration of Helsinki and obtained Ethics Committee approval from the Institutional Review Board of Beijing Tongren Hospital, Capital Medical University (TRECKY2019-146). There was full communication with all participants' parents or legal guardians and written informed con- sent was obtained from all of them. The study completed the clinical trial registration with the number of ChiCTR1900026693.

Lhasa, with an area of $29,518 \mathrm{~km}^{2}$, is one of the main population centers of the Tibetan people in Tibet Autonomous Region, China. According to the Chinese census data in 2010, the Tibetan population of Lhasa is 429,104 , accounting for $76.7 \%$ of the total population of Lhasa. And the 9-year compulsory education is well implemented in Lhasa, with an enrollment rate of $99.7 \%$. Therefore, school-based sampling is highly representative for the sameage population.

Students who had been living in Lhasa for at least half a year and would continue to live there for 5 years or more were eligible. Individuals suffering from mental illness or other medical conditions that were unable to cooperate with the baseline survey and follow-up would be excluded. Stratified cluster sampling was used in LCES. Based on the evaluation of local government, 27 out of the 28 elementary schools in Lhasa available joining the study were stratified into 3 levels. Finally, 1,942 grade 1 students of 7 elementary schools were randomly sampled by stratified cluster sampling.

\section{Examinations}

The flow diagram of the LCES examination is shown in Figure 1. Basic systematic examinations were composed of height, weight, body circumference, oxygen saturation measured by digital fingertip pulse oximeter (YX301; YUWELL, Jiangsu, China), heart rate and blood pressure measured by digital automatic blood pressure monitor (HK-808; HSH, Shenzhen, China).

Presenting visual acuity (VA), wearing present correction if spectacles were carried, was measured monocularly (right followed by left eye) using Lea Symbols 3-m Set charts (250300; Goodlite, Elgin, IL, USA) at $3 \mathrm{~m}$. All the subjects were taught to identify the optotypes using the matching card before the examination. The contralateral eye was occluded with an eye patch for monocular measurements. The subjects were asked to read the numbers on the right edge of each line (starting from the top line) until they made a mistake, and then were turned attention to the 2 lines above the line where they made the initial mistake. The subjects were asked to read each number on successive lines until 3 or more errors occurred. The last line attempted, combined with the number of errors made on it and previous lines, was used to calculate a letter-by-letter logMAR VA score [17].

Ocular alignment was assessed using the Hirschberg light reflex test, alternate cover test, and cover-uncover test. Cover tests were performed with fixation targets at both distance $(6 \mathrm{~m})$ and near $(33$ $\mathrm{cm})$. The presence of strabismus and its characteristics, type, and size were also recorded.

Objective refraction for every participant was measured before and after cycloplegia using an autorefractor (KR-800; Topcon, Tokyo, Japan). Three readings of sphero-cylindrical autorefraction were averaged. The cycloplegia was administered with 2 drops of $1 \%$ cyclopentolate (Alcon) and 1 drop of tropicamide/phenylephrine hydrochloride (Santen, Japan) 5 min apart. A third drop of cyclopentolate would be administered if pupillary light reflex was still present or the pupil size was less than $6.0 \mathrm{~mm} 30$ min later [18]. Subjective refraction was performed on all participants with VA < $20 / 20$ in either eye using a trial frame placed and adjusted on the participant's face. Autorefractor readings were used as the starting point and refinements of sphere, cylinder and cylinder axis were performed until the best-corrected distant VA was obtained by trained optometrists [19]. 


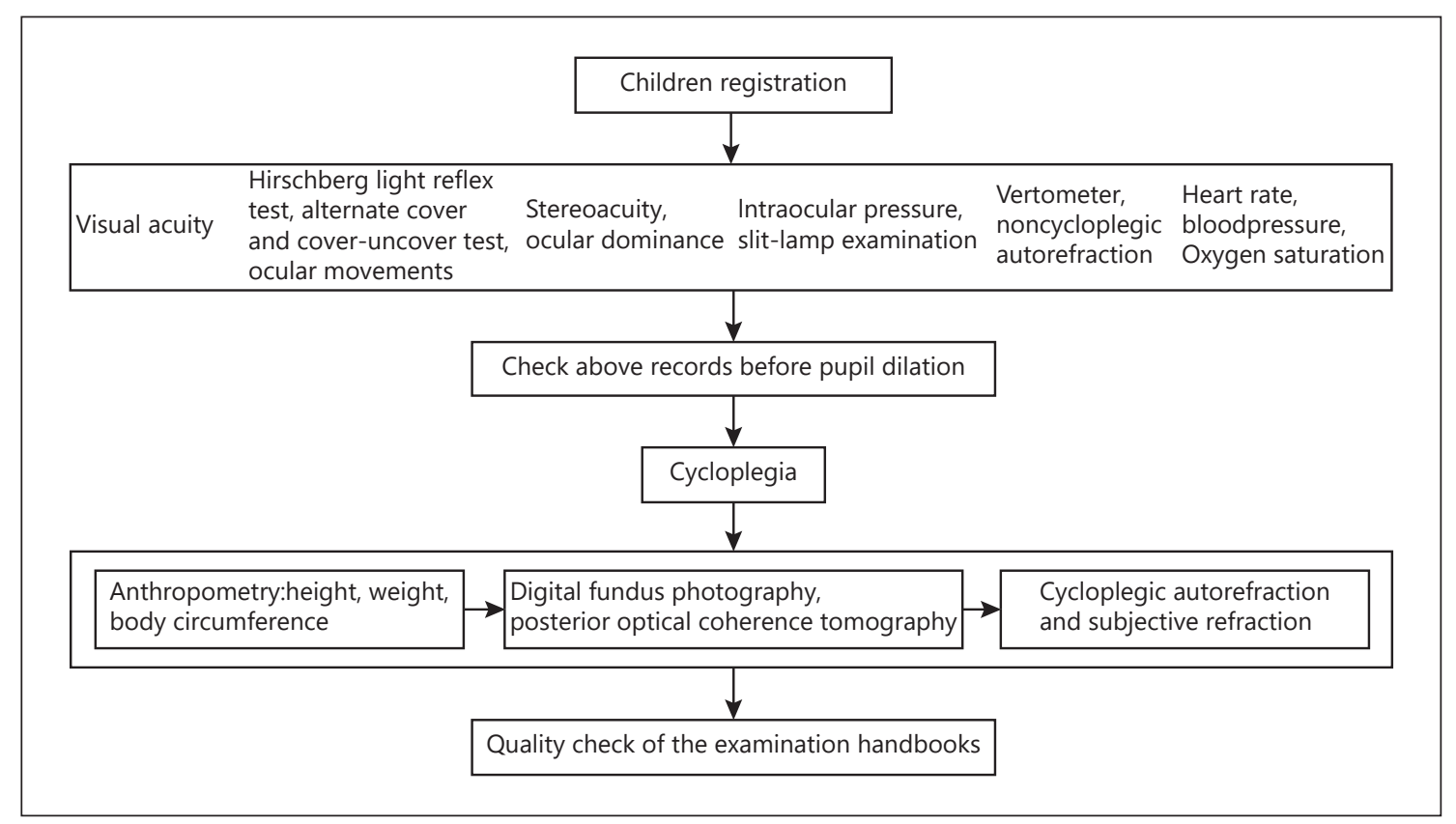

Fig. 1. Flow diagram of the LCES examination. VA, visual acuity.

Other eye examination included stereopsis screening (S0001; STEREO, California, USA), ocular dominance, slit lamp examination (SL-3G; Topcon, Tokyo, Japan), and intraocular pressure (CT-800; Topcon, Tokyo, Japan). Optical coherence tomography and retinal photographs (3D OCT-1; Topcon, Tokyo, Japan) were taken and assessed to exclude retinal anomalies or pathologies.

The standard operating procedures were produced for staff training during the study. All examinations were required to be performed according to procedures. Experienced bilingual staff were employed to decrease language and cultural barriers. Two ophthalmologists (FJ and CWW) worked on site to ensure the procedures were strictly performed.

\section{Questionnaire}

The questionnaire used in LCES mainly consulted the ACES [18] and the Sydney Childhood Eye Study (SCES) [20]. In general, the questionnaire contained information including birth history, living and reading habits, history of diagnosis and treatment, and parents' information such as education, pregnancy history, and other potentially required information. The questionnaires were completed by parents or legal guardians who had been fully informed.

\section{Definitions}

Unilateral amblyopia was defined as the interocular difference of 2 lines or more with the best-corrected visual acuity (BCVA) $\leq 20 / 32$ ( $\geq 0.2 \log$ MAR) in the worse eye and met at least one of the following risk factors: strabismus or previous surgery of strabismus, anisometropia ( $\geq 1.00 \mathrm{D}$ spherical equivalent (SE) anisohyperopia or $\geq 3.00 \mathrm{D}$ SE anisomyopia or $\geq 1.50 \mathrm{D}$ anisoastigmatism), and past or present obstacle of visual axis (e.g., cataract, ptosis, corneal opacity). Bilateral amblyopia was defined as BCVA of both

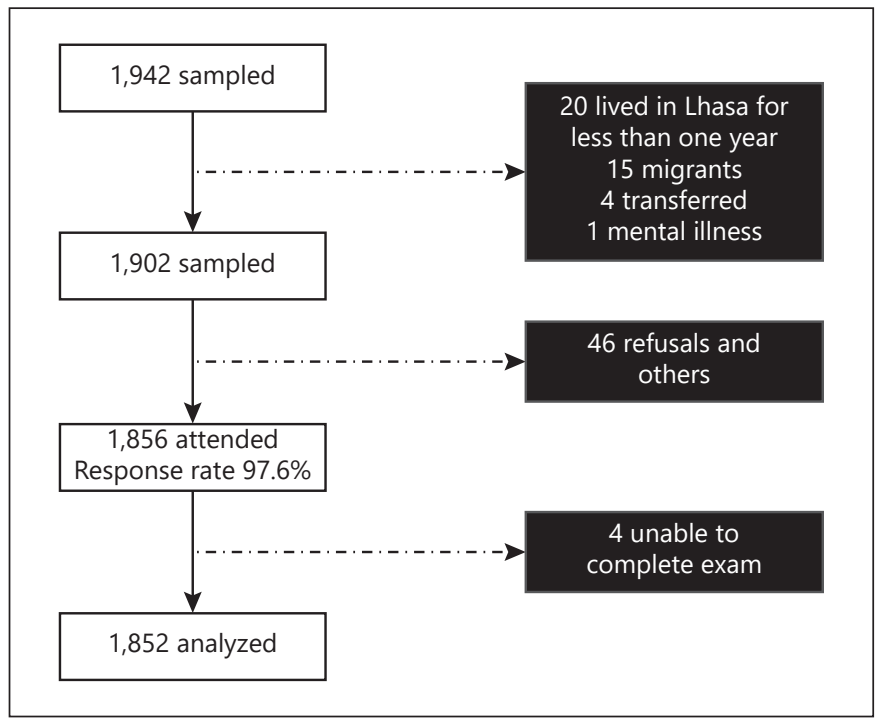

Fig. 2. Flow chart of the enrollment process in the study.

eyes $<20 / 40$ ( $>0.3 \operatorname{logMAR})$ with bilateral ametropia $(\geq 4.00 \mathrm{D} \mathrm{SE}$ hyperopia or $\leq-6.00 \mathrm{D} \mathrm{SE}$ myopia or $\geq 2.50 \mathrm{D}$ astigmatism absolute value) or with past or present bilateral obstacle of visual axis [21].

Myopia and hyperopia were defined as $\leq-0.50 \mathrm{D}$ SE and $\geq+2.00$ D SE, respectively, in 1 or both eyes. Astigmatism was defined as cylindrical refraction absolute value $\geq 1.00 \mathrm{D}$. Strabismus was defined as constant or intermittent heterotropia of any magnitude at distance or near fixation [12]. 
Table 1. Prevalence of amblyopia by different causes in LCES

\begin{tabular}{lrlrl}
\hline Amblyopia type & $N^{\mathrm{a}}$ & Prevalence $^{\mathrm{a}}(95 \% \mathrm{CI}), \%$ & $N^{\mathrm{b}}$ & Prevalence $^{\mathrm{b}}(95 \% \mathrm{CI}), \%$ \\
\hline Unilateral & 23 & $1.24(0.74,1.75)$ & 26 & $1.40(0.87,1.94)$ \\
Strabismic & 4 & $0.20(0.00,0.43)$ & 4 & $0.22(0.00,0.43)$ \\
Anisometropic & 16 & $0.86(0.44,1.29)$ & 19 & $1.03(0.57,1.48)$ \\
Strabismic + anisometropic & 2 & $0.11(-0.04,0.26)$ & 2 & $0.11(-0.04,0.26)$ \\
Deprivation & 1 & $0.05(-0.05,0.16)$ & 1 & $0.05(-0.05,0.16)$ \\
Bilateral & 11 & $0.59(0.24,0.94)$ & 17 & $0.92(0.48,1.35)$ \\
Ametropic & 9 & $0.49(0.17,0.80)$ & 15 & $0.81(0.40,1.22)$ \\
Deprivation + ametropic & 1 & $0.05(-0.05,0.16)$ & 1 & $0.05(-0.05,0.16)$ \\
Other causes (nystagmus) & 1 & $0.05(-0.05,0.16)$ & 1 & $0.05(-0.05,0.16)$ \\
\hline
\end{tabular}

LCES, Lhasa Childhood Eye Study; CI, confidence interval. ${ }^{a} n$ and prevalence were estimated by the definition used by MEPEDS (Multiethnic Paediatric Eye Disease Study). ${ }^{b} n$ and prevalence were estimated by the definition used by PEDIG (Pediatric Eye Disease Investigator Group).

\section{Feedback and Treatment}

Every student who completed the examination got a detailed report with diagnostic information and therapic recommendations in a month, provided by 2 experienced pediatric ophthalmologists.

\section{Statistical Analysis}

All statistical analyses were performed using SAS statistical software (version 9.4, SAS Inc., Cary, NC, USA) and two-sided $p<0.05$ was considered statistically significant. Values were presented as mean \pm standard deviation for continuous variables and percentage for categorical variables. Prevalence was calculated as the percent of participants with amblyopia among all the participants evaluated. Difference of gender and prematurity between 2 groups was compared using $\chi^{2}$ test. And difference in age, BMI, and oxygen saturation between 2 groups was compared using $t$ test. Multivariate logistic regression models were used to evaluate the effect of each refractive risk factor while adjusting for each other. All of the confidence intervals (CIs) are given as 95\% CIs.

\section{Results}

\section{Subject}

Forty of 1,942 students did not meet the inclusion criteria. 1,856 of the eligible 1,902 individuals participated in the examinations ( $97.58 \%$ response rate) and 4 of them failed to complete the autorefraction examinations. Finally 1,852 students completed all the amblyopia-related examinations. The flow chart of enrollment was shown in Figure 2. The mean age of participants was $6.83 \pm 0.46$ years (ranging from 5.89 to 10.32 years). Only 2 children were $<6$ years old. There were 1,271 and 555 children aged $6-7$ and $7-8$ respectively, accounting for $98.60 \%$ of the total. Nineteen children were between the age of $8-9$, while 5 above the age of 9 . Therefore, the age of partici- pants was relatively concentrated on 6-8 years. 980 students (52.92\%) were males, and 872 were females (47.08\%). 1,758 of the 1,852 students $(94.92 \%)$ were Tibetans, while $85(4.59 \%)$ were Han and $9(0.49 \%)$ were of other ethnicities.

\section{Prevalence of Amblyopia}

Thirty-four of 1,852 students met the definition of amblyopia (1.84\%, 95\% CI: 1.22-2.45\%), and another 3 students were suspected. All of the 34 definite students with amblyopia were Tibetans and included 19 girls and 15 boys. There was no significant difference in age $(t=-0.89$, $p=0.38)$, gender $\left(\chi^{2}=1.08, p=0.30\right)$, premature $\left(\chi^{2}=\right.$ $1.05, p=0.31), \operatorname{BMI}(t=-0.22, p=0.83)$, and oxygen saturation $(t=-1.56, p=0.13)$ between students with or without amblyopia. The mean BCVA of amblyopic eyes was $0.54 \pm 0.22 \log$ MAR ranging from 0.2 to $1.0 \log$ MAR.

As shown in Table 1, by the definition used in the MEPEDS (Multiethnic Pediatric Eye Disease Study) [21], unilateral amblyopia was diagnosed in 23 children (1.24\%, 95\% CI: 0.74-1.75\%), and bilateral amblyopia was diagnosed in 11 students $(0.59 \%, 95 \%$ CI $0.24-0.94 \%)$. Of 11 children with bilateral amblyopia, 9 were ametropic, 1 had the deprivation factor induced by ptosis in the right eye and ametropic factor in the left eye, and 1 had congenital nystagmus without any other eye disease detected. Twenty-three children with unilateral amblyopia contained 16 anisometropic amblyopia, 4 strabismic amblyopia, 1 stimulus deprivation amblyopia caused by congenital cataract, and 2 mixed amblyopia including strabismus and anisometropia.

In addition, 3 children without strabismus and deprivation factor met the VA criteria for unilateral amblyopia, 
Fig. 3. Distribution of SER in amblyopic eyes and nonamblyopic right eyes. SER, spherical equivalent refraction.
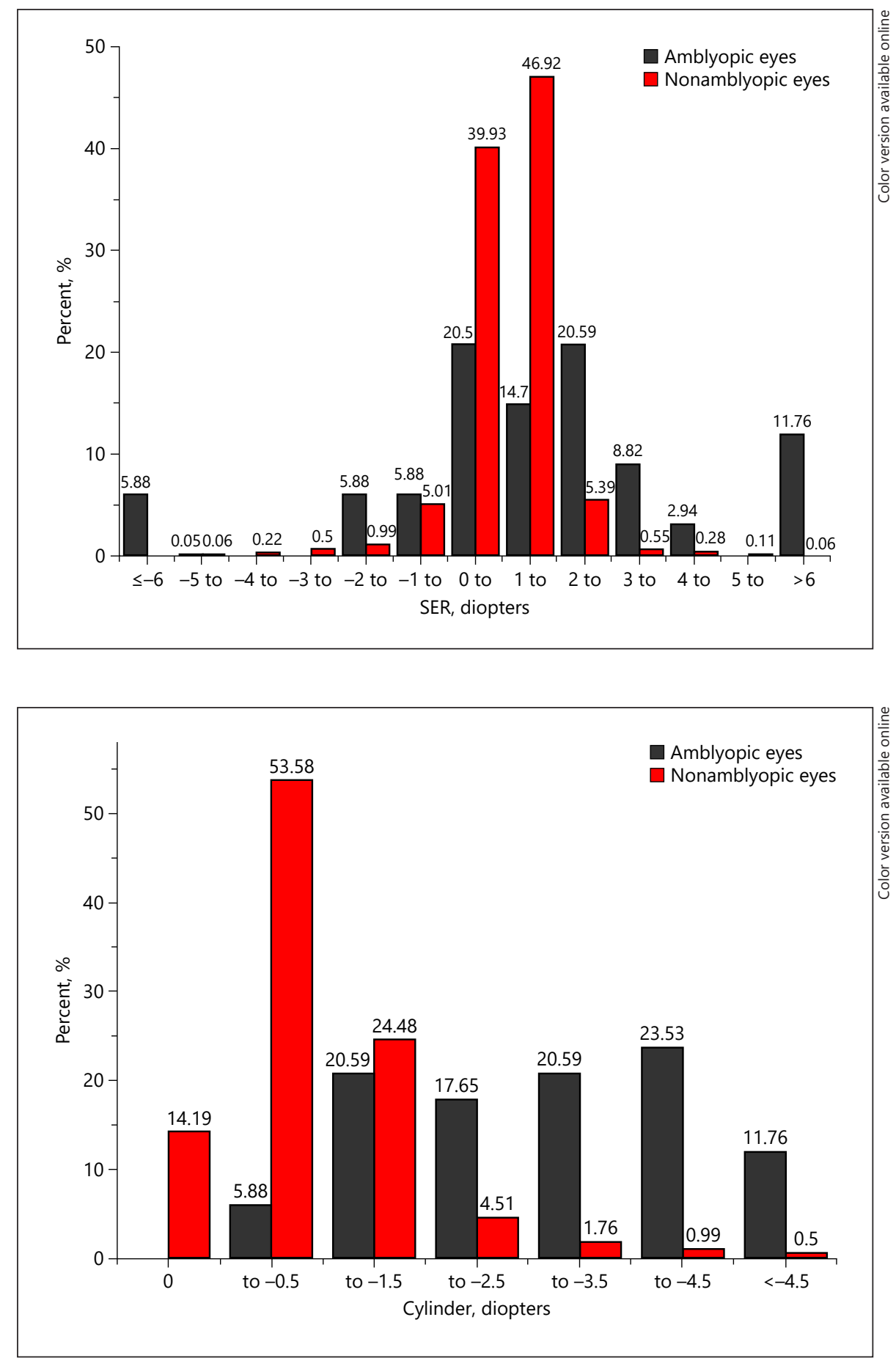

Fig. 4. Distribution of cylinder refraction in amblyopic eyes and nonamblyopic right eyes. but the difference in astigmatism between 2 eyes was 1.25 $\mathrm{D}$, missing the critical value $1.5 \mathrm{D}$ in the diagnosis criteria of amblyopia. If these 3 children were considered as amblyopic, the prevalence of amblyopia increased to $1.99 \%$.

The definition of amblyopia varied among different studies. In order to better explain the prevalence under different criteria, the prevalence based on the PEDIG (Pediatric Eye Disease Investigator Group) criteria and MEPEDS was presented in Table 1. PEDIG defined the unilateral amblyopia as interocular difference of 2 lines or more and bilateal amblyopia as BCVA of both eyes $\leq 20 / 40$ $(\geq 0.3 \log M A R)[22]$, and according to the above defini- 
Table 2. Multivariate analysis for refractive factors of amblyopia ${ }^{a}$

\begin{tabular}{|c|c|c|c|c|}
\hline Refractive errors & $N$ & Amblyopia, N (\%) & OR (95\% CI) & $p$ value \\
\hline \multicolumn{5}{|l|}{ SE refractive error, D } \\
\hline$\leq-0.50$ & 75 & $7(9.33)$ & $2.55(0.83-7.90)$ & 0.1039 \\
\hline$>-0.50$ and $<+2.00$ & 1,615 & $11(0.68)$ & Ref & \\
\hline$\geq+2.00$ & 162 & $16(9.88)$ & $8.22(3.42-19.72)$ & $<0.0001$ \\
\hline \multicolumn{5}{|l|}{ Astigmatism, D } \\
\hline$>-1$ and $\leq 0$ & 1,467 & $9(0.61)$ & Ref & \\
\hline$>-2$ and $\leq-1$ & 256 & $8(3.13)$ & $2.21(0.76-6.45)$ & 0.1470 \\
\hline$\leq-2$ & 129 & $17(13.18)$ & $6.76(2.56-17.85)$ & 0.0001 \\
\hline \multicolumn{5}{|l|}{ SE anisometropia, D } \\
\hline$\geq 0$ to $<+0.50$ & 1,631 & $10(0.61)$ & Ref & \\
\hline$\geq+0.50$ to $<+1.00$ & 171 & $9(5.26)$ & $3.95(1.44-10.79)$ & 0.0075 \\
\hline$\geq+1.00$ & 50 & $15(30.00)$ & $21.90(8.24-58.18)$ & $<0.0001$ \\
\hline
\end{tabular}

OR, odds ratio; CI, confidence interval; SE, spherical equivalent. ${ }^{a}$ Adjusted for all the other refractive risk factors in a multivariate logistic regression model. The more ametropic eyes in unilateral amblyopia and less ametropic eyes in bilateral amblyopia were analyzed.

tion, a total of 43 children were diagnosed as amblyopia with the prevalence of $2.32 \%$ (95\% CI: 1.64-3.01\%). Unilateral amblyopia was diagnosed in 26 children $(1.40 \%$, 95\% CI: 0.87-1.94\%), including 19 anisometropic, 4 strabismic, 1 stimulus deprivation caused by congenital cataract, and 2 mixed amblyopia containing strabismus and anisometropia. Bilateral amblyopia was diagnosed in 17 children (0.92\%, 95\% CI 0.48-1.35\%) including 15 ametropic, 1 mixed amblyopia with the deprivation factor induced by ptosis in the right eye and ametropic factor in the left eye, and 1 congenital nystagmus without any other eye disease detected.

\section{Refractive Error and Amblyopia}

The average of the absolute value of the SE refraction of amblyopic eyes was $2.70 \pm 2.33 \mathrm{D}$ (ranging from -9.00 to $+6.75 \mathrm{D}$ ) compared to $1.17 \pm 0.65 \mathrm{D}$ (ranging from -4.375 to $+8.25 \mathrm{D})$ in nonamblyopic eyes $(t=-3.82$, $p=$ 0.0006 ). The mean cylinder refraction of amblyopic eyes was $-2.15 \pm 1.52 \mathrm{D}$ (ranging from -5.75 to 0 ) compared to $-0.63 \pm 0.74 \mathrm{D}$ (ranging from -6.5 to $0 \mathrm{D}$ ) in nonamblyopic eyes $(t=-5.80, p<0.0001)$. Figures 3 and 4 illustrated the SE and cylinder refraction distribution of the amblyopic and nonamblyopic eyes. Figure 3 showed SE of nonamblyopic eyes mainly concentrated from -1.00 to $+2.00 \mathrm{D}$, while SE of the amblyopic eyes was relatively dispersed from $\leq-6.00 \mathrm{D}$ to $\geq+6.00 \mathrm{D}$. The cylinder refraction of amblyopic eyes concentrated mainly from the moderate to high degree and nonamblyopic eyes mainly concentrated from mild to moderate degree.

Prevalence and Risk Factors of Amblyopia in Tibetan School Children
The association between refractive errors and prevalence of amblyopia was shown in Table 2 by multivariate logistic regression analysis. Among 34 amblyopic students, 7 were diagnosed with myopia, 16 with hyperopia, 25 with astigmatism, and 24 with anisometropia of $\mathrm{SE}$ equal to or greater than $0.5 \mathrm{D}$. Amblyopia was associated with hyperopia $(\geq+2.00 \mathrm{D}$, OR $8.22,95 \%$ CI $3.42-$ $19.72)$, astigmatism $(\leq-2.00 \mathrm{D}$, OR $6.76,95 \%$ CI $2.56-$ $17.85)$, and anisometropia $(\geq+0.50$ to $<+1.00 \mathrm{D}$, OR $3.95,95 \%$ CI $1.44-10.79 ; \geq+1.00$ D, OR $21.90,95 \%$ CI 8.24-58.18).

According to the American Association of Pediatric Ophthalmology (AAPOS)-specified threshold levels [23, 24], the prevalence of the refractive risk factors was shown and compared with that in MEPEDS in Table 3. The prevalence of anisometropia $>1.50 \mathrm{D}$, hyperopia $>3.50 \mathrm{D}$, astigmatism $>1.50 \mathrm{D}$, and myopia $>3.00 \mathrm{D}$ in the LCES was respectively $1.13,1.24,11.50$, and $0.43 \%$, compared with $2.0,8.4,10.4$, and $1.0 \%$ reported in the MEPEDS [25].

\section{Strabismus and Amblyopia}

There were a total of 68 children diagnosed as manifest strabismus in the study with the prevalence of $3.7 \%$. Fourty-five of the 68 strabismic children were diagnosed as intermittent exotropia and other 23 were diagnosed as constant strabismus. Of the 6 strabismic amblyopic children including 2 mixed with anisometropia, 4 were diagnosed as constant esotropia with the mean deviation angle of $42.50 \pm 8.29$ prism diopters, 1 was diagnosed as 
Table 3. The prevalence of the refractive risk factors in LCES and MEPEDS by AAPOS levels

\begin{tabular}{llllr}
\hline Risk factor & AAPOS & \multicolumn{2}{l}{ LCES } & $\begin{array}{l}\text { MEPEDS, } \\
\%\end{array}$ \\
\cline { 2 - 4 } & criteria, D & $\begin{array}{l}\text { estimated prev- } \\
\text { alence, } \%\end{array}$ & \\
\hline Anisometropia & $>1.50$ & 1.2 & $1.13 \%(21)$ & 2.0 \\
Hyperopia & $>3.50$ & 6 & $1.24 \%(23)$ & 8.4 \\
Astigmatism & $>1.50$ & 9 & $11.50 \%(213)$ & 10.4 \\
Myopia & $>3.00$ & 0.5 & $0.43 \%(8)$ & 1.0 \\
\hline
\end{tabular}

AAPOS, the American Association of Pediatric Ophthalmology [23, 24]; MEPEDS, Multi-ethnic Paediatric Eye Disease Study [25]; LCES, Lhasa Childhood Eye Study. micro-esotropia with the deviation angle of 10 prism diopters and 1 was diagnosed as constant exotropia with the deviation angle of 40 prism diopters.

\section{History and Treatment of Amblyopia}

Six students who met our criteria of amblyopia had a previous diagnosis and other 28 were newly diagnosed during LCES. Four of the six students had been treated with spectacles or monocular occlusion therapy reported by their parents and other 2 students did not have any therapy ever.

In addition, 8 students with related risk factors had previous diagnosis and treatment of amblyopia reported by their parents. But they were not shown to be amblyopic in this study by our criteria. All of the 8 students had significant isoametropia which consisted of 2 astigmatism, 2 hyperopia, and 4 astigmatism-mixed hyperopia. If the 8 students had not received treatment, they would most likely been amblyopic. And the prevalence of amblyopia would increase to $2.27 \%$. Of all the 45 patients including the 34 newly diagnosed, 8 ever diagnosed as amblyopia and 3 suspected cases who met the sub-astigmatism threshold, 12 were wearing spectacles.

\section{Discussion}

LCES is a school-based cohort study to investigate ocular diseases of school-age children in the Tibetan plateau areas in China where few studies related had been developed. It is well established and characterized with high response rate $(97.58 \%)$ and high cycloplegic percentage (99.84\%) in the participants. Lhasa, accounting for more than $1 / 5$ population of Tibet, is the political, cultural, economic, and educational center of Tibet. It is of representative significance to observe the incidence and factors associated with the etiology of amblyopia as well as other ocular diseases for Tibetan children. LCES is also an important complement to other population studies in China, as well as the world because of the distinct environment, living habits, and cultural characteristics in Tibet.

In the present study the prevalence of amblyopia was $1.84 \%$, relatively higher compared to most other studies in Asia. Two meta-analysis articles estimated the pooled global prevalence rate of amblyopia was 1.44 and $1.75 \%$ respectively $[4,26]$. Geographically, amblyopia was more prevalent in Europe $(2.90 \%, 95 \%$ CI $2.20-3.83 \%)$ and North America $(2.41 \%, 95 \%$ CI $1.18-4.88 \%)$ than in Asia (1.09\%, 95\% CI $0.89-1.33 \%)$ and Africa $(0.72 \%, 95 \%$ CI $0.26-1.98 \%$ ) [4]. The prevalence is influenced by factors of age, region, environment, ethnicity, diagnosis criteria, and so on. Different definitions can have significant impact on amblyopia prevalence estimate. As reported, prevalence was highest when a BCVA $\geq 2$ line difference criteria was used $(2.99 \%, 95 \%$ CI $1.89-4.68 \%)$ and lowest when the BCVA $\leq 20 / 40$ criteria was used $(0.76 \%, 95 \%$ CI $0.51-1.14 \%$ ) [4]. We compared the prevalence of amblyopia estimated by different definitions used by the MEPEDS and PEDIG. According to the PEDIG criteria, the prevalence of either unilateral or bilateral amblyopia was higher than that used in MEPEDS. The unilateral amblyopic children diagnosed by the PEDIG criteria included 3 sub-astigmatism threshold cases who were not diagnosed by the MEPEDS criteria. And the bilateral amblyopic children diagnosed by the PEDIG criteria included 6 cases with the VA equalled to 20/40 which did not meet the criteria by MEPEDS (VA < 20/40). Therefore, minor difference in the definition would impact on the prevalence. In order to be comparable we summarized several previous studies in Table 4 with the same standard amblyopia definition from MEPEDS [21, 27]. Compared with other studies on children close in age and of different nationalities, the prevalence in the Tibetan plateau region was relatively higher $[12,15]$. Pi et al. [28] reported a 
Table 4. Prevalence of amblyopia in different studies using the MEPEDS criteria

\begin{tabular}{|c|c|c|c|c|c|c|}
\hline Study & Country & Year & $N$ & Age & Ethnicity & Prevalence, \% \\
\hline MEPEDS & USA & 2008 & 3,350 & 30-72 months & $\begin{array}{l}\text { African American }(1,663) \\
\text { Hispanic/Latino }(1,687)\end{array}$ & $\begin{array}{l}1.50 \\
2.60\end{array}$ \\
\hline MEPEDS & USA & 2013 & 1,883 & 30-72 months & $\begin{array}{l}\text { Asian American (938) } \\
\text { Non-Hispanic White (945) }\end{array}$ & $\begin{array}{l}1.81 \\
1.81\end{array}$ \\
\hline STARS & Singapore & 2010 & 1,682 & 30-72 months & Singaporean Chinese & 1.19 \\
\hline SPEDS & Australia & 2012 & 1,422 & $30-72$ months & $\begin{array}{l}\text { Multiethnic } \\
\text { (Predominantly White) }\end{array}$ & 1.90 \\
\hline ACES & China & 2014 & 2,893 & $6-9$ years & Chinese Han & 1.00 \\
\hline HCES & China & 2019 & 7,713 & $30-83$ months & Chinese Han & 1.09 \\
\hline LCES & China & 2019 & 1,852 & $5-10$ years & Chinese Tibetan & 1.84 \\
\hline
\end{tabular}

MEPEDS, Multi-ethnic Paediatric Eye Disease Study [21, 26]; BPEDS, Baltimore Paediatric Eye Disease Study [7]; STARS, Strabismus, Amblyopia, and Refractive Error in Singaporean Children Study [28]; SPEDS, Sydney Paediatric Eye Disease Study [6]; ACES, Anyang Childhood Eye Study [12]; NPVP, Nanjing Paediatric Vision Project; YPEDS, Yuhuatai Paediatric Eye Disease Study [13]; MMPS, Mojiang Myopia Progression Study [15]; HCES, Hunan Childhood Eye Study [16]; LCES, Lhasa Childhood Eye Study.

higher prevalence rate of $1.88 \%$ in Western China than our study. But the diagnosis criteria of amblyopia was different from ours which defined the BCVA as $\leq 20 / 25$ or worse.

The higher prevalence in our study might be related to distinct factors of ethnicity, environment, and economics. Participants of our study were most native Tibetan (94.92\%) who lived on the high altitude area of 3,650 m where the diet habit and environment such as climate, light, and pressure were obviously different from plateau. All of above could lead to the physical difference besides the ethnic factor. In economically underdeveloped areas the popularity of vision screening is relatively low. The risk factors of amblyopia cannot be detected in time which could lead to delay in the early diagnosis and treatment of amblyopia. Lhasa is relatively remote, and healthcare is not quite universal. Only 6 of 34 students with amblyopia had a previous diagnosis. Among the 6 students, only 4 accepted treatments with spectacles or monocular occlusion therapy. And another 2 students did not get any therapy ever even when their parents knew their

Prevalence and Risk Factors of Amblyopia in Tibetan School Children situation of poor vision. The rate of diagnosis and treatment in $\operatorname{LCES}(17.65,11.76 \%)$ was lower compared to ACES which was also developed during students with close age in plain area of China $(22.22,14.81 \%)$ [12]. Besides, 18 students had amblyopia history reported by parents in questionnaire but did not meet the criteria of diagnosis in our examination. Among the 18 students 10 might be misdiagnosed because they had normal VA without any amblyopic risk factors and treatment history. And other 8 students who had related risk factors and previous therapy history did not meet the VA criteria. Therefore, standard screening and intervention as well as the improvement of parents understanding of amblyopia at remote areas are necessary for increasing cure rate and reducing unnecessary time and economic loss. All of the students with amblyopia got the feedback of detailed diagnostic information and therapic recommendations after the examination, provided by 2 experienced pediatric ophthalmologists in the group.

In this study, gender was not significantly associated with amblyopia prevalence which was in agreement with 
a number of previous studies $[29,30]$. Because the age of participants in the study was relatively concentrated, it was difficult to identity that age was associated with the prevalence or not. Besides, there was no significant difference in prematurity, BMI, and oxygen saturation between students with or without amblyopia.

The study showed anisometropia and ametropia were major causes to the prevalence of amblyopia. Eighteen students with anisometropic amblyopia (52.94\%) consisted of 11 anisoastigmatism, 3 anisohyperopia, 1 anisomyopia, and 3 mixed anisometropia (2 anisoastigmatism-mixed anisohyperopia and 1 anisoastigmatismmixed anisomyopia) according to the diagnosis criteria. Some studies reported anisometropia of $1.0 \mathrm{D}$ or more was associated significantly with increased odds of amblyopia $[6,14]$. Our study demonstrated anisometropia of $0.5-1.0 \mathrm{D}$ was also associated significantly with the increased risk of amblyopia which was consistent with other previous report [31]. Refractive errors which included hyperopia of $2.00 \mathrm{D}$ or more and astigmatism of $-2.00 \mathrm{D}$ or less were also significant risk factors for amblyopia. Myopia was not identified as significantly associated with increased odds of amblyopia in our study. It might be because myopia was less common in younger children (4.05\%).

According to the AAPOS-specified threshold levels, the prevalence of astigmatism $>1.50 \mathrm{D}$ in the LCES (11.5\%) was higher than that in the MEPEDS (10.4\%). And the prevalence of anisometropia $>1.50 \mathrm{D}(1.13 \%)$, myopia $>3.00 \mathrm{D}(0.43 \%)$, and hyperopia $>3.50 \mathrm{D}(1.24 \%)$ was lower than that in the MEPEDS $(2.0,1.0,8.4 \%$ respectively). The significant difference for the prevalence of hyperopia $>3.50 \mathrm{D}$ between the 2 studies might be due to the different age of participants which was mainly concentrated on 6-8 years in LCES and 36-60 months in MEPEDS.

The proportion of strabismic amblyopia was relatively less in LCES compared with some studies [9], although the prevalence of manifest strabismus was higher than that reported in MEPEDS [25]. The reason might be the strabismus detected in LCES was most intermittent which usually had minimal impact on VA. And esotropia strabismus was also an important cause for amblyopia in LCES.

Our study also had some limitations. First, the study was school based rather than population based. This could have biased our findings either towards overestimation or underestimation. But the high enrollment rate for primary school-age children in Lhasa (99.7\%) and the high response rate in LCES (97.58\%) reduced the bias.
Second, amblyopia history and treatment in questionnaires were self-reported from the parents, which might be potentially inaccurate. In the follow-up study, we will further improve the quality of the questionnaires.

In conclusion the study is representative to observe the incidence and factors associated with the etiology of amblyopia for Tibetan children. We find the prevalence of amblyopia is $1.84 \%$ in grade 1 students in Lhasa which is higher than that of many other ethnic populations in China previously reported. Anisometropia, hyperopia, and astigmatism are the major risk factors of amblyopia.

\section{Acknowledgements}

We are grateful for the support from the Lhasa government and Beijing Hoson Foundation for helping organizing the survey.

\section{Statement of Ethics}

The study adhered to the Declaration of Helsinki and obtained Ethics Committee approval from the Institutional Review Board of Beijing Tongren Hospital, Capital Medical University (TRECKY2019-146). The written informed consent was obtained from the parents or guardians.

\section{Conflict of Interest Statement}

The authors have no conflicts of interest to declare.

\section{Funding Sources}

The study was supported by High-Level Health Technical Talent Training Program of Beijing Municipal Health Bureau under Grant (2015-3-023), Beijing Municipal Science \& Technology Commission under Grant (Z171100001017066), and Capital Health Development Special Fund of China-Major Project under Grant (SF-2018-1-2051).

\section{Author Contributions}

J.F. and W.W.C. initiated the study design. Z.J.M., W.W.C., Y.Y., W.D., and H.S. prepared the consent form. All authors are involved in the research process and data collation. L.L. assisted in collation and analysis of the data. Z.J.M. and J.F. drafted and finalized the study protocol. 


\section{References}

1 Carlton J, Karnon J, Czoski-Murray C, Smith KJ, Marr J. The clinical effectiveness and costeffectiveness of screening programmes for amblyopia and strabismus in children up to the age of 4-5 years: a systematic review and economic evaluation. Health Technol Assess. 2008 Jun; 12(25):iii,

2 Carlton J, Kaltenthaler E. Amblyopia and quality of life: a systematic review. Eye. 2011 Apr;25(4):403-13.

3 Felius J, Chandler DL, Holmes JM, Chu RH, Cole SR, Hill M, et al. Evaluating the burden of amblyopia treatment from the parent and child's perspective. J AAPOS. 2010 Oct;14(5): 389-95.

4 Fu Z, Hong H, Su Z, Lou B, Pan CW, Liu H. Global prevalence of amblyopia and disease burden projections through 2040: a systematic review and meta-analysis. Br J Ophthalmol. 2020 Aug;104(8):1164-70.

5 Attebo K, Mitchell P, Cumming R, Smith W, Jolly N, Sparkes R. Prevalence and causes of amblyopia in an adult population. Ophthalmology. 1998 Jan;105(1):154-9.

6 Pai AS, Rose KA, Leone JF, Sharbini S, Burlutsky G, Varma R, et al. Amblyopia prevalence and risk factors in Australian preschool children. Ophthalmology. 2012 Jan;119(1): 138-44.

7 Friedman DS, Repka MX, Katz J, Giordano L, Ibironke J, Hawse P, et al. Prevalence of amblyopia and strabismus in white and African American children aged 6 through 71 months the Baltimore pediatric eye disease study. Ophthalmology. 2009 Nov;116(11):2128-2.

8 Robaei D, Kifley A, Rose KA, Mitchell P. Impact of amblyopia on vision at age 12 years: findings from a population-based study. Eye. 2008 Apr;22(4):496-502.

9 Robaei D, Rose KA, Ojaimi E, Kifley A, Martin FJ, Mitchell P. Causes and associations of amblyopia in a population-based sample of 6-year-old Australian children. Arch Ophthalmol. 2006 Jun;124(6):878-84.

10 Elflein HM, Fresenius S, Lamparter J, Pitz S, Pfeiffer N, Binder $\mathrm{H}$, et al. The prevalence of amblyopia in Germany: data from the prospective, population-based Gutenberg health study. Dtsch Arztebl Int. 2015 May;112(19): 338-44.

11 Høeg TB, Moldow B, Ellervik C, Klemp K, Erngaard D, la Cour M, et al. Danish rural eye study: the association of preschool vision screening with the prevalence of amblyopia. Acta Ophthalmol. 2015 Jun;93(4):322-9.
12 Fu J, Li SM, Li SY, Li JL, Li H, Zhu BD, et al. Prevalence, causes and associations of amblyopia in year 1 students in Central China: the Anyang childhood eye study (ACES). Graefes Arch Clin Exp Ophthalmol. 2014 Jan;252(1): 137-43.

13 Chen X, Fu Z, Yu J, Ding H, Bai J, Chen J, et al. Prevalence of amblyopia and strabismus in Eastern China: results from screening of preschool children aged 36-72 months. Br J Ophthalmol. 2016 Apr;100(4):515-9.

14 Huang D, Chen X, Zhu H, Ding H, Bai J, Chen $\mathrm{J}$, et al. Prevalence of amblyopia and its association with refraction in Chinese preschool children aged 36-48 months. Br J Ophthalmol. 2018 Jun;102(6):767-71.

15 Zhu H, Pan C, Sun Q, Huang D, Fu Z, Wang $J$, et al. Prevalence of amblyopia and strabismus in Hani school children in rural southwest China: a cross-sectional study. BMJ Open. 2019 Feb;9(2):e025441.

16 Li YP, Zhou MW, Forster SH, Chen SY, Qi X, Zhang HM, et al. Prevalence of amblyopia among preschool children in central south China. Int J Ophthalmol. 2019 May;12(5): 820-5.

17 Chen W, Fu J, Meng Z, Li L, Su H, Dai W, et al. Lhasa childhood eye study: the rationale, methodology, and baseline data of a 5 year follow-up of school-based cohort study in the Tibetan plateau region of Southwest China. BMC Ophthalmol. 2020 Jun;20(1):250.

18 Li SM, Liu LR, Li SY, Ji YZ, Fu J, Wang Y, et al. Design, methodology and baseline data of a school-based cohort study in Central China: the Anyang childhood eye study. Ophthalmic Epidemiol. 2013 Dec;20(6):348-59.

19 Yuan BL, Friedman DS, Wong TY, Wang FH, Duan XR, Xiao HY, et al. Rationale, design, methodology, and baseline data of a population-based study in rural China: the Handan eye study. Ophthalmic Epidemiol. 2009 MarApr;16(2):115-27.

20 Ojaimi E, Rose KA, Smith W, Morgan IG, Martin FJ, Mitchell P. Methods for a population-based study of myopia and other eye conditions in school children: the Sydney myopia study. Ophthalmic Epidemiol. 2005 Feb; 12(1):59-69.
21 Multi-Ethnic Pediatric Eye Disease Study Group. Prevalence of amblyopia and strabismus in African American and Hispanic children ages 6 to 72 months the multi-ethnic pediatric eye disease study. Ophthalmology. 2008 Jul;115(7):1229-36.e1.

22 Wallace DK, Repka MX, Lee KA, Melia M, Christiansen SP, Morse CL, et al. Amblyopia preferred practice pattern ${ }^{\circledast}$. Ophthalmology. 2018 Jan;125(1):P105-42.

23 Donahue S, Arnold R, Ruben JB. Preschool vision screening: what should we be detecting and how should we report it? Uniform guidelines for reporting results from studies of preschool vision screening. J AAPOS. 2003 Oct; 7(5):314-6.

24 Arnold RW. Amblyopia risk factor prevalence. J Pediatr Ophthalmol Strabismus. 2013 Jul-Aug;50(4):213-7.

25 Varma R. Amblyopia refractive risk factors (letter reply). Ophthalmol. 2012 Jun;119(6): 1283-4.

26 Hashemi H, MSc RP, Yekta A, Bostamzad P Aghamirsalim M, MSc SS, et al. Global and regional estimates of prevalence of amblyopia: a systematic review and meta-analysis. Strabismus. 2018 Dec;30:1-16.

27 McKean-Cowdin R, Cotter SA, Tarczy-Hornoch K, Wen G, Kim J, Borchert M, et al. Prevalence of amblyopia or strabismus in asian and non-Hispanic white preschool children: multi-ethnic pediatric eye disease study. Ophthalmology. 2013 Oct;120(10):2117-24.

28 Pi LH, Chen L, Liu Q, Ke N, Fang J, Zhang S, et al. Prevalence of eye diseases and causes of visual impairment in school-aged children in Western China. J Epidemiol. 2012 Nov;22(1): 37-44.

29 Chia A, Dirani M, Chan YH, Gazzard G, Au Eong KG, Selvaraj P, et al. Prevalence of amblyopia and strabismus in young singaporean chinese children. Invest Ophthalmol Vis Sci. 2010 Jul;51(7):3411-7.

30 Mocanu V, Horhat R. Prevalence and risk factors of amblyopia among refractive errors in an Eastern European population. Medicina. 2018 Mar;54(1):6.

31 Tarczy-Hornoch K, Varma R, Cotter SA, McKean-Cowdin R, Lin JH, Borchert MS, et al. Risk factors for decreased visual acuity in preschool children: the multi-ethnic pediatric eye disease and Baltimore pediatric eye disease studies. Ophthalmology. 2011 Nov; 118(11):2262-73. 